L'appareil à influencer de Tausk et les concepts de la structure et de l'automatisme dans la psychiatrie et la psychanalyse de son temps

\title{
Yorgos Dimitriadis
}

\section{Introduction}

Le célèbre article de Victor Tausk « La genèse de la machine à influencer » [35] - dont il a fait la présentation durant deux sessions de la société psychanalytique de Vienne, les 6 et 30 janvier 1919 [1] porte sur l'évolution et la structure d'une forme de délire d'influence dans la schizophrénie, dans lequel un certain type de schizophrènes se plaignent d'être persécutés. Le délire de persécution avec appareil à influencer serait, selon Tausk [36], une variante très rare de délire, et son étude tire des conclusions, surtout d'un exemple unique que l'auteur considère comme atypique. Mais, l'aspect atypique de cas permettrait, selon cet auteur, de chercher les origines et les conditions d'apparition des phénomènes. « Il est, dit-il ([36], p.178), admissible de tirer, des formes aberrantes ou des variantes, des conclusions sur la structure de la forme commune...C'est seulement lorsqu'un objet peutêtre différente, ne fût-ce qu'une seule fois, qu'on trouve l'occasion de vérifier les raisons pour lesquelles, d'habitude, il apparaissait invariablement identique ». Pour introduire la genèse de l'appareil à influencer, Tausk reconnaît, d'abord, des cas de patients qui se plaignent - simplement - des sensations de transformation, ou des sentiments d'étrangeté physique ou psychique, mais sans pour cela en chercher la cause dans une puissance hostile ou étrangère. Il s'agirait 
d'un sentiment d'altération, provoqué par la stase libidinale au niveau de l'organe (hypocondrie). Celui-ci pourrait, par la suite, devenir sentiment d'aliénation, suscité par le refus, opposé par le moi, de l'organe malade - qui se trouve ainsi exclu du reste du moi « sain ». Lequel, à son tour, peut déboucher sur le sentiment de persécution (il introduit le terme "paranoia somatica» à cet égard), issue de la projection des modifications pathologiques dans le monde extérieur. Deux cas de figures sont possibles, ici, selon l'auteur : soit, attribuer leur origine à une puissance étrangère hostile; soit, construire un appareil à influencer pour réunir, en un ensemble, les projections vers l'extérieur de tous les organes malades et, de manière privilégiée, les organes génitaux. Cette machine, qui constitue l'appareil à influencer, présente des images aux malades, produit et dérobe leurs pensées, produit des actions motrices dans le corps du malade et produit des sensations variées, électriques, magnétiques, atmosphériques. Il peut déclencher, aussi, éruptions cutanées, furoncles et autres processus morbides somatiques. Ainsi, la machine à influencer serait une manifestation plus tardive, qui permettrait au patient de donner un sens aux sensations primaires de transformation, lesquelles n'avaient pas de contenu idéique auparavant. D’après cette conception, dit-il ([36], p.180), «la machine à influencer est créée par le besoin de causalité immanent à l'homme », quoique tous les patients ne cherchent pas la cause de ces sentiments dans une puissance hostile ou étrangère.

Selon Tausk [36], le processus de la genèse de l'appareil à influencer ramène au stade évolutif, au cours duquel la trouvaille de 
l'objet se faisait encore au niveau du corps propre, celui-ci étant considéré ainsi comme le monde extérieur. La projection du corps dans cet appareil - serait alors une défense contre une position libidinale, correspondant à celle de la fin du développement fœetal et au début du développement extra-utérin. Cette projection pathologique provient ainsi d'une accumulation de libido narcissique, analogue à la libido primaire, mais intempestive, régressive ou résiduelle, identique au narcissisme inné. La régression de la libido est due au défaut d'évolution de certaines pulsions durant l'enfance. De ce fait, quand un conflit psychique survient durant la vie adulte, des réactions d'autoguérison peuvent apparaître, mais aussi, la régression des fonctions qui avaient évolué normalement. Ces dernières régressent pour atteindre le niveau de celles qui n'avaient pas évolué.

\section{La machine à influencer et la question de la structure en}

\section{psychiatrie}

D'abord, nous allons tenter de situer cet article, véritable chant du cygne de son auteur - car il s'est suicidé peu après, le 3 juillet 1919 - dans le cadre de la psychiatrie de son époque. Selon la classification de Georges Lanteri-Laura [25], il fait partie - chronologiquement - de la période que cet auteur a qualifiée de «deuxième paradigme de la psychiatrie » (dans le sens du paradigme de T. Kuhn [18]). Plus précisément, Lanteri-Laura considérait que ce paradigme commençait en 1854, année de la publication de l'article de Jean-Paul Falret [13], «De la non-existence de la monomanie», qui combattait une conception de la maladie mentale, en tant que maladie unique, et 
finissait en 1926. Durant cette deuxième période, en effet, les phrénologues essayaient de décrire les maladies mentales - quant à leurs diverses espèces- selon la sémiologie, l'évolution et l'étiologie de chacune d'entre elles. Ce paradigme aurait succédé à un premier paradigme, lancé en 1793 par Philippe Pinel, le premier à avoir intégré la folie - qu'il appelait «aliénation »- dans le cadre de la médecine, en tant que maladie mentale (au singulier). Nous ajoutons ici que la conception d'une évolution de la folie, d'un stade initial au stade suivant, existait déjà pour des auteurs comme Guislain ${ }^{1}$ (fondateur de la psychiatrie belge), qui avait décrit le concept de la phénalgie initiale (1952) : sentiment d'angoisse diffus et de douleur morale qui, par la suite, crée des représentations délirantes conformes à lui. En France, dans la seconde moitié du $19^{\text {ème }}$ siècle, Charles Lasègue [26] et Valentin Magnan [27], ont décrit - chacun à sa manière - le délire chronique : Lasègue $(1852)^{2}$ comme un état qui évolue en deux phases, tandis que Magnan ${ }^{3}$ (1893) comme un état qui évolue systématiquement en quatre phases. Le troisième paradigme de la psychiatrie, toujours selon Lanteri-Laura [25], aurait commencé en 1926, année du congrès de Lausanne - où Eugen Bleuler a fait sa fameuse conférence sur la

\footnotetext{
${ }^{1}$ Cf. Paul Bercherie ([3], p.60-61).

2 Le délire de persécution à évolution progressive: malaise indéfinissable puis, systématisation progressive $\mathrm{du}$ délire de persécution.

${ }^{3}$ Le délire chronique à évolution systématique en quatre phases : incubation, délire de persécution, mégalomanie, et démence.
} 
schizophrénie - et finirait en 1977 avec la mort d'Henri Ey. Durant ce dernier paradigme, divers psychiatres - souvent influencés par la théorie de la forme et la-dite «neurologie globaliste » - essayèrent d'isoler l'élément caractéristique de chaque entité psychopathologique, dans une tentative de concevoir chaque état psychopathologique comme une entité, dans son ensemble (im Ganzheit) ${ }^{4}$. Ainsi, tandis que dans le deuxième paradigme l'importance était donnée à la description et à l'évolution, dans le troisième paradigme on donnerait une importance particulière à la structure, c'est à dire à l'élément sous-jacent présent, malgré la diversité apparente des tableaux cliniques.

Dans l'article de Tausk [36] «La genèse de l'appareil à influencer », au-delà des remarques de l'auteur sur l'évolution du délire, nous constatons une telle focalisation sur sa structure. Car Tausk présente avec clarté la distinction entre ce qui est cliniquement superficiel (moins important et secondaire), et le processus unique - qui est invariablement présent. Cette reproduction constante concerne, d'une part, l'évolution de l'état clinique d'un cas particulier - la façon

${ }^{4}$ La distinction, faite par Eugen Bleuler [6], entre symptômes fondamentaux et accessoires de la schizophrénie, « la perte vitale avec la réalité » d'Eugène Minkowski [30], « la fuite des idées » de Ludwig Binswanger [5] et la dissolution de la conscience et de la personnalité dans les psychoses aigues et chroniques, respectivement dans la théorie organodynamique d'Henri Ey [12], feraient partie de cette tendance de la psychiatrie. 
dont il se présente durant ses diverses phases ${ }^{5}$ - et, d'autre part, le fait que les divers cas ne présentent que partiellement le processus commun sous-jacent. Seulement rarement, un cas va présenter tous les stades du processus, mais, quand cela se produit, s'ouvre - à cette occasion - une fenêtre qui va nous laisser apercevoir les rouages de tous les autres cas, qui présentent le même processus, mais de manière dissimulée et partielle ; c'est à dire que, selon Tausk, ce constat va nous permettre de dire que tous ces cas ont la même structure et appartiennent à la même série. Ainsi ce travail de Tausk, au delà de la description de l'évolution du délire est conforme à la recherche de l'élément sous-jacent présent, malgré la diversité apparente des tableaux cliniques qui caractérisait le troisième paradigme, selon Lanteri-Laura. Ce n'est pas certainement un effet du hasard, que soit donné - précisément durant cette phase de la psychiatrie - une importance particulière aux phénomènes dits automatiques de la psychose, c'est à dire des phénomènes qui se répètent comme une sorte de structure épurée du délire. Mais, c'est aussi durant cette même période que Freud va développer ses théories sur des phénomènes de répétition en rapport avec l'inconscient ${ }^{6}$.

\footnotetext{
${ }^{5}$ Et un seul clinicien aurait la possibilité d'observer toutes ces phases encore plus rarement.

${ }^{6}$ Pour aller vite : en rapport avec - entre autres - ses concepts de « symbole mnémonique », de «l'effet après coup », de répétition/remémoration » et de «compulsion de répétition »,
} 
Par ailleurs, la façon de Tausk d'aborder le délire ressemble à l'analyse mécanique de Gaëtan de Clérambault $[8]^{7}$ - et, en même temps, comme nous verrons, la dépasse - durant cette même période (1919-1927), avec son concept du petit automatisme mental, mais aussi avec la structure de l'érotomanie, qui avait - selon de Clérambault trois phases dans les cas purs (de l'espoir, du dépit, puis, de la rancune); mais, cette typicité ne s'exprimait que rarement, comme c'était le cas du processus de délire avec l'appareil à influencer de Tausk. Pour des raisons de conformité avec la thématique de la «xénopathie », nous allons nous limiter, ici, à une description succincte de l'automatisme mental, en sa forme élémentaire- à savoir, le-dit petit automatisme mental. Celui-ci consiste en une série de phénomènes que de Clérambault avait divisés en positifs et négatifs. Les phénomènes «positifs » sont des phénomènes qui se manifestent comme parasitaires du train habituel de la pensée, comme l'écho de la pensée. Les phénomènes «négatifs" sont des phénomènes comme l'inhibition et la confusion. Enfin, il existe des phénomènes « mixtes »: par exemple, le sentiment d'irréalité et la fausse reconnaissance de personnes et de situations. Tous ces phénomènes ont en commun le fait qu'ils sont sans contenu idéique, c'est à dire qu'ils sont « anidéiques » et neutres, d'un point de vue affectif. Pour de Clérambault, cet aspect confirme leur origine mécanique et, par conséquent, ceci lui permettait

Wiederholungszwang, traduit par Lacan comme «automatisme de répétition ».

${ }^{7}$ Lacan l'a désigné en 66 [19] comme son seul maître en psychiatrie. 
de constater la parenté des «psychoses à base d'automatisme » avec les psychoses d'origine toxique et organique. Tandis que ces phénomènes élémentaires apparaissent brutalement au début de la psychose, le délire est une construction autour de ces phénomènes, qui se manifestent dans la conscience et « exigent» une interprétation de la part du sujet. De ce fait, quand le délire entre dans le tableau clinique, en tant que «superstructure », la psychose est déjà bien établie.

Lacan a trouvé, dans les travaux de de Clérambault, une façon d'aborder les phénomènes basaux de la psychose à la limite de la signification. Il a écrit ([19], p.65) en hommage à de Clérambault : " Son automatisme mental, avec son idéologie mécanistique de métaphore, bien critiquable assurément, nous paraît, dans ses prises du texte subjectif, plus proche de ce qui peut se construire d'une analyse structurale, qu'aucun effet clinique dans la psychiatrie française ». Nous avons vu, plus haut, que ce même type de processus - avec des phénomènes «anidéiques » et neutres - était présent, durant la même période, dans la conception de Tausk [36], quand il postulait la possibilité d'existence de sentiments d'altération - mais, sans sentiment d'aliénation - ou des sentiments d'aliénation - mais, sans sentiments de persécution - dans la psychose.

\section{La machine à influencer et le structuralisme en psychanalyse}

Trente sept ans plus tard - dans son séminaire sur les psychoses Lacan ([21], p.284) a développé cette idée de façon explicitement structuraliste, quand il a stipulé «qu'il faut rattacher le noyau de la psychose à un rapport du sujet au signifiant sous son aspect le plus 
formel, sous son aspect de signifiant pur, et que tout ce qui se construit là autour n'est que réactions d'affect au phénomène premier, le rapport au signifiant ». Mais Tausk [36] avait déjà fait un bout de trajet dans ce sens. Ainsi, nous sommes d'accord avec Claude Rabant [31] quand ce dernier soutient que Tausk utilise une méthode structuraliste avant son ère et, dirait-on, encore plus que de Clérambault ne le faisait ${ }^{8}$. Car, Lacan [21] et, comme nous l'avons vu, dans une certaine mesure Tausk - à contrario de Clérambault - considéraient le délire, non pas comme un résultat d'induction (d'enkystement des phénomènes élémentaires dans la personnalité), mais comme le résultat de la même «force structurante » qui produit les phénomènes élémentaires. Nous citons Lacan ([21], p.28) : «c'est toujours la même force structurante qui est à l'œuvre dans le délire, qu'on le considère dans une de ses parties ou dans sa totalité ». Et il ajoute « Le délire n'est pas déduit, il en reproduit la même force constituante, il est, lui même, un phénomène élémentaire $»$.

Néanmoins, la différence entre la conception de Lacan et celle de Tausk est considérable : elle correspond à la différence entre la psychanalyse du temps de Tausk, c'est à dire, de la période de la première topique, et celle de la période où Lacan étudiait les travaux de Freud à l'aide de la linguistique structuraliste de Ferdinand de Saussure et de l'anthropologie structurale de Claude Levi-Strauss, dans les années '50. Tausk tentait d'appliquer la théorie freudienne sur le narcissisme - en tant que stade évolutif de la libido (avec son éventuelle

\footnotetext{
${ }^{8}$ Cf. aussi Hulak (17).
} 
stase) - l'identification à l'objet de l'amour et le mécanisme de projection dans le cas de Melle Natalia A., ainsi que son extension possible dans d'autres cas de délire avec «appareil à influencer». A noter le caractère pionnier du travail de Tausk, durant une période où les psychanalystes évitaient d'entreprendre des cures analytiques avec des patients psychotiques. Tausk [36] va au delà de la théorie freudienne de cette période, et souligne «l'identification au corps», qui est initialement perçu comme étranger, comme morcelé -disjecta membra ${ }^{9}$ - avant d'être perçu comme une entité - à travers l'identification au corps propre, comme une «psyché du moi »- de ce qui existait avant, en tant qu'investissement autoérotique du corps propre. C'est cette identification qui va servir de support à la construction de la machine, qui va agir comme une projection (et en même temps une aliénation) des parties du corps et des stimulations relatives ${ }^{10}$ à celles-ci. Sa théorie - « de l'identification au corps propre » - lui permet de faire avancer d'un pas la théorie psychanalytique de la psychose. De surcroît, il prend une distance audacieuse avec la théorie freudienne, quand il questionne le choix exclusivement homosexuel du

${ }^{9}$ En référence implicite au terme « disiecti membra poetae » du poète lyrique romain Horace [16] Cette expression nous rappelle le stade du miroir et le morcèlement de l'image du corps dans la psychose, que Lacan [20] va développer plus tard.

${ }^{10}$ Le modèle serait, selon Tausk [36], l'érection du pénis qui impressionne le garçon, en constatant qu'il ne peut contrôler une partie de son corps. 
persécuteur dans la paranoïa - en soulignant que ce qui importe n'est pas l'opposition entre les sexes, mais l'opposition entre libido objectale et narcissique. On pourrait le situer, quelque part, entre la théorie freudienne sur la psychose de Schreber [14] (le juge psychotique dont Freud a commenté in extenso son autobiographie) et la première théorie lacanienne sur la psychose - telle qu'il l'a développée dans son troisième séminaire [21], Lacan, comme Freud [14] avant lui, considérait que la causalité de la psychose repose sur un mécanisme autre que le refoulement (qui concerne la névrose), auquel il va donner le nom de «forclusion », terme qu'il a employé pour traduire le mot freudien de Verwerfung. Néanmoins, il a considéré que ce qui est forclos dans la psychose n'est pas l'homosexualité, mais la fonction paternelle (le signifiant Nom-du-Père) ${ }^{11}$. Tandis que Freud a tenté d'expliquer la psychose par l'Edipe, Lacan [21] a considéré la psychose comme un processus en dehors de l'Edipe. Freud s'est basé sur la grammaire - le déni, l'inversion et la rejection de la phrase « Moi (un homme) aime lui (un homme) ([14], p.299); tandis que Lacan a fait usage de la linguistique structuraliste.

${ }^{11}$ Selon Lacan, le rapport de passivité et l'absence de limitation par la fonction phallique - entre le sujet psychotique homme et la jouissance est «féminisant», et c'est à ce type de relation qu'il attribue « l'homosexualité », ou plutôt « le transsexualisme », dans la psychose chez l'homme, et non pas - comme Freud - à une tendance homosexuelle que le psychotique repousserait. 
Pour Lacan, le névrosé est représenté par le signifiant dans le but de se préserver des excitations de pulsions, c'est à dire de la jouissance de l'Autre. Le psychotique, quant à lui, ne disposant pas de fonction signifiante, est forcé de « compléter l'Autre ». Schreber est astreint de compléter les messages incomplets de Dieu ; Nathalia A. (la patiente de Tausk) complétait la machine à influencer, avec des pièces qui devenaient - au fur et à mesure - de moins en moins anthropomorphiques, et qui, de cette façon, localisaient la jouissance quelque part - à une distance de sécurité du sujet psychotique. Par conséquent, Tausk avait perçu la fonction de «condensateur de jouissance », terme que Lacan ([22], p.90) a employé à propos du «symptôme» psychotique. Plus tard, d'autres auteurs ont tenté de démontrer comment la conception - ou même la construction - des objets-machines par des sujets autistes peut fonctionner comme compensation, c'est à dire, comme une auto-guérison qui vise la concentration de la jouissance ; comme dans le cas de Joey, autiste que Bruno Bettelheim [4] a commenté et dans des cas d'autisme de JeanClaude Maleval [29]. Ce dernier soutient que les autistes sont parfois capables de construire un Autre de compensation, qui est fait de « signes », lesquels leur permettent de gérer l'Autre du code linguistique, comme un objet qu'ils peuvent manipuler (mémoire photographique, calcul de chiffres ou de dates, conception des machines spécifiques etc) ${ }^{12}$.

\footnotetext{
${ }^{12}$ Ludivine Beillard-Robert [2] distingue la machine autistique qui entretient une relation symbiotique avec le sujet là où la machine
} 


\section{Remarques conclusives}

Nous avons vu que l'article de Tausk [36] sur l'appareil à influencer a été conçu au moment où la psychiatrie, après une période de «réflexion» sur l'évolution des délires, a tenté de synthétiser le processus délirant, à travers le concept de la structure sous-jacente ; et les phénomènes d'automatisme sont une telle structure. C'est à cette même période que se fait la découverte de l'inconscient freudien, qui sera reprise et fructifiée par le structuralisme en psychanalyse ${ }^{13}$.

Nous avons vu l'aspect pionnier des travaux de De Clérambault en psychiatrie, mais sa découverte ${ }^{14}$ est allée de pair avec une « dénégation » de l'inconscient. Car nous notons, encore ici $[9,11]$, que les théories organicistes en psychiatrie sur l'automatisme mental, tendent à méconnaître la possibilité de l'inconscient de produire des phénomènes de ce genre et que les psychotiques, à leur manière, tendent aussi - par le caractère xénopathique de ces phénomènes - à méconnaître le contenu de leur inconscient ${ }^{15}$. Comme Nathalia A., qui

psychotique entretient une relation d'influence malveillante.

${ }^{13}$ Lacan, dans Télévision [23], va jusqu'à utiliser le terme inconscient (et le terme langage), comme synonyme du terme structure.

14 Nous empruntons ce terme à Christophe Chaperot [7].

${ }^{15}$ Nous faisons surtout référence à la théorie de De Clérambault qui, néanmoins, dans son dernier texte de 1934 ([8], p.610-612), sur le délire autoconstruit, n'a pas omis la possibilité de causes non organiques, en faisant la distinction entre délire explicatif et délire autoconstruit. Il y a ajouté qu'une causalité affective de ces phénomènes ne 
refusait de se reconnaître soi-même (on dirait : sa subjectivité), dans la machine anthropomorphe qu'elle a inventée.

Dans ce sens, nous considérons la contribution de Tausk sur la machine à influencer, comme un travail pionnier de l'aspect « créatif» de l'automatisme mental. Tausk a effectué ce travail dans le même sillage que Freud (inspiré par la philosophie romantique de son temps ${ }^{16}$ ) considérant les formations de l'inconscient comme une forme d'automatisme créatif. Nous avons vu que Tausk était même en avance sur certains aspects de l'automatisme chez les psychotiques par rapport à son maître. Sur ces aspects, Tausk est allé dans le même sens avec Lacan qui, quelques décennies plus tard, inspiré par la linguistique et l'anthropologie structurale, mais aussi, par la cybernétique de son époque, a pu allé bien plus loin; ceci en considérant l'inconscient structuré comme un langage, avec des qualités d'automate - à travers son interprétation de la théorie d'Aristote sur la fortune ( $\tau \dot{\chi} \chi \eta)$ et l'automaton $(\alpha v \tau o ́ \mu \alpha \tau o)^{17}$.

Enfin, une certaine période tend à créer des conditions propices à la découverte simultanée d'une même idée ${ }^{18}$ par plusieurs chercheurs, qui vont fréquemment s'accuser mutuellement de vol d'idées. Freud et Tausk se sont adressé réciproquement des accusations de ce genre [32], devrait pas être exclue. Cf. aussi sur ce sujet l'article de Remi Tévissen [37].

\footnotetext{
${ }^{16}$ Cf. Lanteri-Laura [24].

${ }^{17} \mathrm{Cf}$. Notre contribution sur ce sujet [10].

${ }^{18}$ Comme une sorte, dirait-on, d'automate.
} 
et de Clérambault avait aussi accusé publiquement Lacan de plagiat, durant une séance de la «Société médico-psychologique » (cf. [28], p.228, [33], p. $124 \&$ [34], p.146), qui lui a retourné la charge. Il est à noter encore ici - car ce fut déjà avancé antérieurement par un autre auteur [15] - qu'il est assez singulier que, dans ces deux cas, ce type d'accusations se soient portées en rapport avec le thème de l'influence sur la pensée. Pourtant, chaque ère est bien plus « responsable» de la naissance d'une idée, qu'un ou plusieurs des ses auteurs.

Alors, durant la période de la découverte du télégraphe, plusieurs théories ont été avancées sur le délire d'influence, mais les délires ont aussi intégré, dans leurs thématiques, des machines, qui ont remplacé le diable, les animaux et l'hypnose de délires des époques antérieures. Si l'inconscient a effectivement structure de langage et possède des qualités d'automate, ce n'est probablement pas dû au hasard que sa découverte ait été faite durant cette période où se passe - suite à la naissance de la science moderne - la révolution industrielle et juste avant l'ère du structuralisme et des théories cybernétiques. Ce point, de la découverte simultanée des automates de toute sorte, mériterait des études supplémentaires.

\section{References}

1. Assoun P.-L. Dictionnaire des œuvres psychanalytiques. Paris : PUF ; 2009. 
2. Beillard-Robert L. Pour une distinction de la machine d'influence et de l'objet autistique, Evolution Pychiatrique 2014; 79 (3) : 527-539.

3. Bercherie P. Les fondements de la clinique. Paris: Navarin ; 1980.

4. Bettelheim B. La forteresse vide, Paris: Gallimard ; 1969.

5. Binswanger L. Sur la fuite des idées. Grenoble: Jérôme Million ; 1968 [1933].

6. Bleuler E. Dementia Praecox ou le groupe de schizophrénies. Paris : EPEL ; 1993 [1913].

7. Chaperot C. Structuralisme, clinique structurale, diagnostic différentiel, névrose-psychose. Paris : L’Harmattan ; 2003.

8. Clérambault, G.-G. (de) Euvres complètes. Paris : Frénésie ; 1987.

9. Dimitriadis, Y. Du délire dogmatique ou spéculatif à l'hypocondrie. Discours Psychanalytique. $1995 ; 13: 21-39$.

10. Dimitriadis, Y. Aristote et les concepts psychanalytiques de «l'effet après coup » et de la répétition. Recherches en psychanalyse, $2009 ; 9$.

11.Dimitriadis, Y. Psychogénèse et organogénèse en psychopathologie : une hypothèse psychanalytique. Paris : L'Harmattan ; 2013.

12. Ey H. Des idées de Jackson à un modèle organo-dynamique en psychiatrie, Paris : L’Harmattan ; 2000 [1938]. 
13. Falret J.-P. De la non-existence de la monomanie. In Des maladies mentales et des asiles d'aliénés. Leçons cliniques et considérations générales. Paris : Sciences en situation ; 1994 [1864].

14. Freud S. Le président Schreber. In Cinq psychanalyses, $18^{\text {ème }}$ éd., Paris : PUF ; 1993[1911] : 263-324.

15. Gillebert J. A partir de Freud et Tausk, "postface" en guise de "préface". In Tausk, V. Oeuvres psychanalytiques. Paris : Payot; $1976: 223-244$.

16. Horace Sermonum Liber Primus (Satires).

17. Hulak F. Délire et mécanisme. In Hulak, F. editor. Pensée psychotique et création de systèmes. Paris : Eres ; 2003.

18. Kuhn T. The structure of scientific revolutions. Chicago : The University of Chicago Press ; 2000 [1962].

19. Lacan, J. De nos antécédents. ; In Écrits. Paris : Seuil ; 1966 [1949] : 73-92.

20. Lacan J. Le stade du miroir. In Écrits. Paris : Seuil, 1966 [1949] : 93-100.

21. Lacan J. Les psychoses, Séminaire III. Paris : Seuil ; 1981 [1957-1958].

22. Lacan J. Le séminaire livre X. L'angoisse, (texte établi par Jacques -Alain Miller), Paris : Seuil ; 2004 [1962-1963].

23. Lacan J. Télévision. Paris : Seuil ; 1974.

24. Lanteri-Laura G. La notion de l'automatisme dans la médicine et dans la psychiatrie moderne. In Grivois, H. Editor. Autonomie et automatisme dans la psychose, Paris : Masson ; 1992 : 7-29. 
25. Lanteri-Laura G. Essai sur les paradigmes de la psychiatrie moderne. Paris : Du temps ; 1998.

26. Lasègue Ch. Du délire de persécution. Archives générales de médecine. $1852 ; 28: 129-150$.

27. Magnan V. Le délire chronique à évolution systématique. Paris : L’Harmattan ; 1998 [1891].

28. Maleval J.-C. G.G. de Clérambault loin de l'oubli ; Evol Psychiatr $2001 ; 66: 223-239$.

29. Maleval J.-C. (2003). De l'objet autistique à la machine, Les suppléances du signe. In Hulak, F. editor. Pensée psychotique et création de systèmes, Paris: Eres : 197-217.

30. Minkowski E. Le temps vécu - Études phénoménologiques et psychopathologiques. Neuchâtel: Delachaux et Niestlé S.A; 2000 [1933].

31. Rabant C. Tausk prélude à Lacan. In Hassoun, J. editor, Freud 1889-1989 : le voyage à Nancy. Nancy : Presses Universitaires de Nancy ; $1990:$ 79-91.

32. Roazen P. Brother animal: the story of Freud and Tausk. New Brunswick: Transaction Publishers ; 1990 [1969].

33. Roudinesco E. La bataille de cent ans. Histoire de la psychanalyse en France. v. II, Paris ; 1986.

34. Sivadon O. Entretien avec F. Caroli. Ornicar, Revue du champ freudien $1986 ; 37: 145-147$. 
35. Tausk V. Über die Entstehung des “Beeinflussungsapparates”. Internationale Zeitschrift für Ärtzliche Psychoanalyse $1919 ; 5$ (1) : 1-33.

36. Tausk V, De la genèse de l' " appareil à influencer » au cours de la schizophrénie. In Oeuvres psychanalytiques ; Paris : Payot ; 1978 : $76-217$.

37. Tévissen R. L'indéterminisme de l'automatisme mental dans sa relation au hasard et à la contingence dans l'histoire de la psychiatrie, Psychiatrie, Sciences Humaines, Neurosciences 2008 : 6 ; 38-53 